\title{
Secagem do açafrão (Curcuma longa L.) em diferentes cortes geométricos
}

\author{
Drying of Curcuma longa L. in different shapes
}

\section{Carlos Alberto Almeida VILELA ${ }^{1 \star}$, Patrícia Oliveira ARTUR ${ }^{2}$}

\section{Resumo}

O processo de secagem de açafrão, atualmente utilizado pela grande maioria dos produtores agrícolas, consiste na exposição deste produto ao ar livre sobre uma lona plástica estendida sobre o solo, utilizando a radiação solar como fonte de energia para a secagem, sem nenhum tipo de aparato técnico para proteção contra a ação do tempo ou de animais. Sob estas condições de secagem, em um ambiente onde praticamente não há nenhum tipo de controle de temperatura e umidade, surgem vários problemas durante o processo, dentre os quais pode-se destacar o tempo demasiado longo para atingir o ponto final desejado, e paralelamente a isto o desenvolvimento de microrganismos nocivos à saúde e a alta deterioração do produto. Por estar sendo utilizado um ambiente ao ar livre e sem proteção contra a ação do tempo, fica também difícil a previsão do tempo de secagem. Desta forma, o período de secagem pode chegar a 25 dias, o qual é muito longo do ponto de vista industrial. Este trabalho apresenta um levantamento das curvas de secagem do açafrão para diferentes condições de temperatura, velocidade do ar e cortes do rizoma. Tais curvas apresentam a influência da casca, do tipo de corte, da temperatura e da velocidade do ar nos tempos de secagem, parâmetros importantes ao desenvolvimento de novos equipamentos e processos de secagem.

Palavras-chave: secagem; desidratação; açafrão; estufa.

\begin{abstract}
The process currently used by most agricultural producers for drying Curcuma longa L. consists in the exposure of the product to atmospheric conditions, on a plastic film on the ground, using solar radiation as energy source for drying, without the use of any kind of apparatus for protection against the weather or animals. Under these drying conditions, in an ambient with practically no temperature and moisture control, various problems can arise, such as an excessively long time to reach desired moisture content, with the concomitant growth of microorganisms harmful to health and high product deterioration. Prediction of drying time is also an information difficult to obtain for this process, because there is no control of drying conditions. The mean time necessary to dry the product can reach twenty five days, which is not industrially interesting. This paper presents Curcuma longa L. drying curves, resultant from experimental drying in a convection oven under different temperature, air velocity and type of cut conditions. It is possible to identify the influence of the peel, type of cut, temperature and air velocity on drying time, important parameters in the development of new drying equipments and processes.

Keywords: food drying; Curcuma longa; convective dryer.
\end{abstract}

\section{Introdução}

A secagem é um dos processos mais tradicionais utilizados para conservação de alimentos, pois o teor de água presente é reduzido e assim o crescimento microbiológico é minimizado. Portanto, é possível aumentar o tempo de conservação sem a presença de aditivos, como também se pode alterar as propriedades organolépticas, tais como textura, cor, sabor, aroma e cor, dando origem a novos produtos, tais como frutas passas, tomates secos, pimenta em pó, alho e cebola em flocos, etc. A retirada de água pode ser realizada através do efeito da temperatura sobre o alimento, quando o processo envolve a transferência de calor ou através de efeito da pressão na superfície.

A utilização da radiação solar como fonte de energia é sem dúvida a forma que requer menor custo de operação. Por sua vez, em alguns casos, torna-se um processo não muito interessante do ponto de vista industrial, devido às variações climáticas, e conseqüentemente, ao longo tempo necessário para atingir o ponto desejado de umidade no alimento. Este tempo pode ser determinado a partir das curvas de secagem dos diversos produtos, que varia em função do tipo de processo, do equipamento utilizado e das condições de secagem. Vários modelos de secadores solares juntamente com resultados experimentais podem ser vistos em Brace Research Institute, MacDonald College of McGill University (1975) e Rossi (1980).

Mesmo sendo utilizada a radiação solar como fonte de energia, a convecção é uma grande responsável pelo fenômeno de secagem, pois será através do gás secante que se dará a transferência de massa do alimento, conforme Figura 1. Tem-se que, no processo de convecção, quanto maior o número de Reynolds envolvido, maiores serão as transferências de calor e massa. Este é o grande motivo para a indústria, que justifica a utilização de

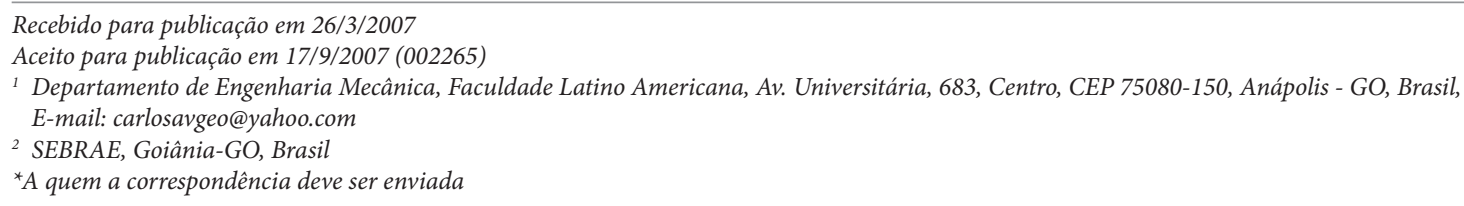




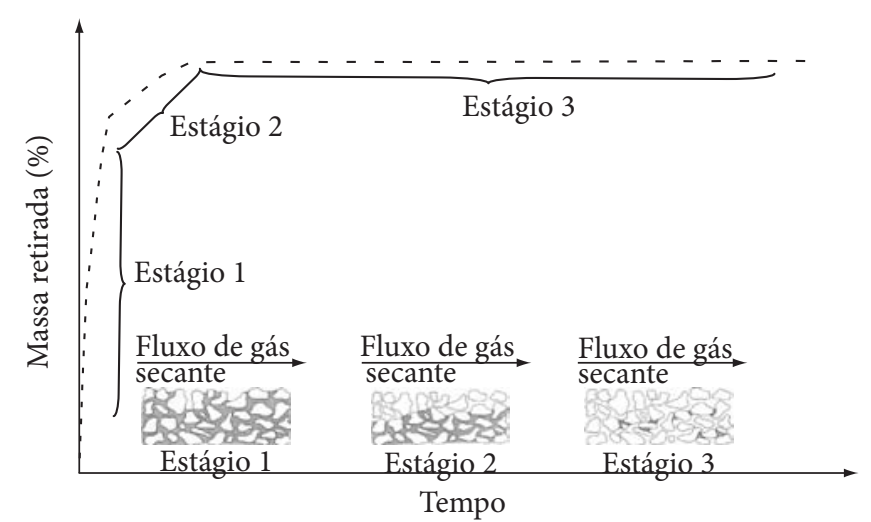

Figura 1. Curva típica da cinética da secagem.

processos artificiais com convecção forçada para diminuir o tempo de secagem. A Figura 1 também mostra os três estágios típicos na cinética da secagem, que são caracterizados pelas diferentes velocidades de retirada de água do produto.

O estágio 1 é caracterizado por um período muito curto de tempo com alta taxa de secagem transiente, e seqüente período de alta taxa de secagem constante. Após o regime permanente ter sido atingido, a temperatura do meio sólido é a mesma temperatura de bulbo úmido do gás secante e permanece estável até iniciar o estágio 2, momento em que o meio atinge a umidade crítica.

O estágio 2 é caracterizado por uma taxa de secagem decrescente, pelo aumento da temperatura do meio sólido e a água é retirada de forma mais lenta.

O estágio 3 é caracterizado pela menor taxa de secagem durante todo o processo, próxima a zero, e a umidade do sólido tende a atingir a umidade de equilíbrio, que é a menor que pode ser conseguida nas condições atuais de secagem.

Novas técnicas têm sido experimentadas e dentre as mais recentes e promissoras está o uso de uma fonte de calor por meio de microondas. Valentini et al. (1998) utilizaram esta técnica para estudos no processo de secagem do grão de milho; Foggiato e Ribani (1999) utilizaram para secagem de feijão; Cabrita et al. (1999) para a secagem de erva-mate e Maskan (2000) utilizou a técnica de secagem por microondas associada à convecção tradicional. De acordo com Maskan (2001), a utilização do microondas mostra-se bastante promissora, pois os resultados obtidos pelos autores mostraram que o tempo de secagem pode ter uma redução significativa em relação aos métodos tradicionais, mas os efeitos da radiação ainda estão sendo avaliados.

Pode-se dizer que uma das principais curvas características de secagem é a de umidade relativa do produto em função do tempo de exposição, também conhecida como cinética da secagem, Figura 1. Esse tipo de curva apresenta a informação de qual o tempo necessário para que o produto atinja uma certa umidade relativa em uma atmosfera com condições específicas de temperatura, umidade, velocidade, pressão, etc. A curva de umidade de equilíbrio, Figura 2, informa qual é o limite de umidade relativa de um produto que pode ser obtido em função da condição da atmosfera secante.

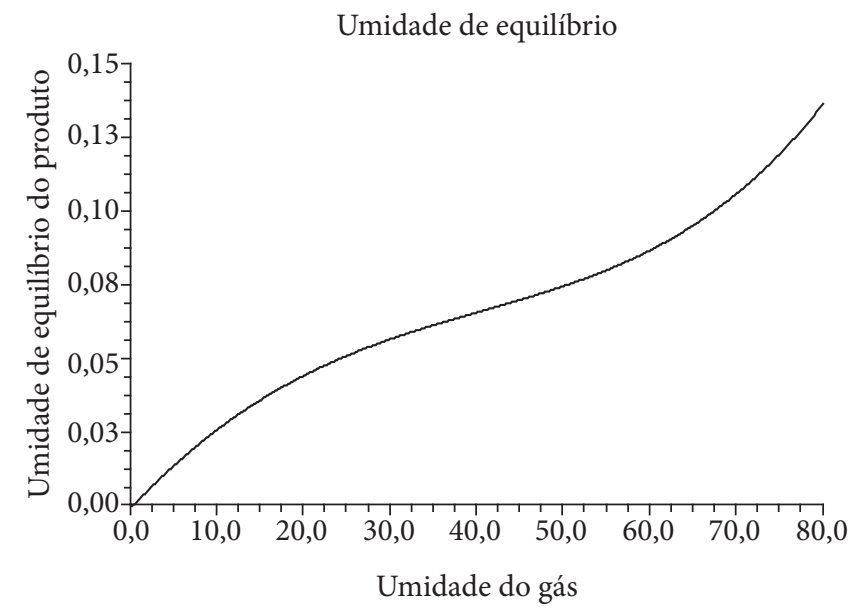

Figura 2. Curva típica de umidade de equilíbrio.

As informações contidas nas curvas de secagem são de fundamental importância para o desenvolvimento de processos e para o dimensionamento de equipamentos. Com elas pode-se estimar o tempo de secagem de certa quantidade de produto e com isso ser feito o planejamento de produção. Com o tempo necessário para a produção estima-se o gasto energético, que refletirá no custo de processamento, que por sua vez influenciará no preço final do produto. No dimensionamento de equipamentos pode-se determinar quais as condições de operação para secagem e com isso a seleção de trocadores de calor, ventiladores, etc.

\section{Material e métodos}

O açafrão (Curcuma longa L.), também conhecido como cúrcuma, gengibre dourado ou açafrão da Índia, é uma espécie originária do sudeste asiático, pertencente à família das Zingiberaceae, planta de pequeno porte que mede aproximadamente $1 \mathrm{~m}$ e largamente cultivada nos países asiáticos, tem sido utilizada há tempos como alimento na forma de condimento ou especiaria, devido ao seu forte sabor e à sua coloração amarelada marcante. Mas a utilização do açafrão não se restringe apenas à alimentação, e hoje está presente em diversas áreas da indústria, medicina, agricultura, etc.

$\mathrm{Na}$ área alimentícia, os rizomas do açafrão depois de secos são moídos e transformados em pó, ou são extraídos deles óleos essenciais, que podem ser utilizados como corantes naturais, antioxidantes e antimicrobianos, qualidades presentes no seu principal composto, a curcumina, Filho et al. (2000). $\mathrm{Na}$ medicina diversas são as utilizações da curcumina, dentre as quais podem-se destacar as propriedades antibacteriana e antiinflamatória, e a ação contra o vírus HIV e contra tumores, Araújo e Leon (2001). Na agricultura o açafrão tem sido bastante utilizado no controle de pragas, como apontado por Peña et al. (2006) A, Peña et al. (2006) B e Kuhn (2006).

Algumas características físico-químicas do açafrão estão apresentadas nas Tabelas 1 e 2 .

Para a realização dos experimentos foi utilizada estufa de convecção forçada, cujas características técnicas estão abaixo relacionadas na Quadro 1. 
Tabela 1. Características físico-químicas do rizoma de açafrão e composição centesimal.

\begin{tabular}{|c|c|c|c|c|}
\hline & $\begin{array}{l}\text { Pruthi } \\
\text { (1980) }\end{array}$ & $\begin{array}{c}\text { Gonvidarajan } \\
(1980)\end{array}$ & $\begin{array}{c}\text { Filho e } \\
\text { Villas-Boas } \\
(1996)\end{array}$ & $\begin{array}{c}\text { Leonel e } \\
\text { Cereda } \\
(2002) \\
\text { (base úmida) }\end{array}$ \\
\hline Umidade & & & 74,70 & 81,23 \\
\hline Proteína & - & $6-11$ & 11,68 & 2,02 \\
\hline Amido & 24,40 & $30-50$ & 35,30 & 8,83 \\
\hline Fibras & - & - & 5,50 & 1,78 \\
\hline Cinzas & 9,00 & $2-6$ & 6,44 & 2,01 \\
\hline $\begin{array}{l}\text { Açúcares } \\
\text { redutores }\end{array}$ & - & - & 1,25 & 0,83 \\
\hline $\begin{array}{l}\text { Açúcares não } \\
\text { redutores }\end{array}$ & - & - & 0,57 & - \\
\hline $\begin{array}{l}\text { Açúcares } \\
\text { solúveis totais }\end{array}$ & - & - & - & 2,02 \\
\hline $\begin{array}{l}\text { Extrato não } \\
\text { nitrogenado }\end{array}$ & - & - & 69,17 & - \\
\hline Extrato etéreo & - & - & 7,20 & - \\
\hline Matéria graxa & & & & 0,91 \\
\hline $\mathrm{pH}$ & - & - & - & 6,54 \\
\hline $\begin{array}{l}\text { Acidez } \\
\text { titulável }\end{array}$ & & & & 10,95 \\
\hline
\end{tabular}

Tabela 2. Concentração de micronutrientes no rizoma do açafrão. Filho et al. (2000).

\begin{tabular}{ccccccccccc}
\hline \multicolumn{11}{c}{ Nutrientes $\left(\mathrm{mg}^{-3} \mathrm{dm}^{-3}\right)$} \\
\hline $\mathrm{N}$ & $\mathrm{P}$ & $\mathrm{K}$ & $\mathrm{Ca}$ & $\mathrm{Mg}$ & $\mathrm{S}$ & $\mathrm{B}$ & $\mathrm{Zn}$ & $\mathrm{Cu}$ & $\mathrm{Mn}$ & $\mathrm{Fe}$ \\
\hline 1,94 & 0,37 & 1,15 & 0,22 & 0,31 & 0,06 & 3,10 & 48,80 & 10,00 & 102,30 & - \\
\hline
\end{tabular}

Quadro 1. Dados técnicos do equipamento.

$\begin{array}{|ll|}\text { Tensão elétrica } & 220 \text { Volts } \\ \text { Potência do ventilador } & 1 / 4 \mathrm{hp} \\ \text { Peso } & 60 \mathrm{~kg} \\ \text { Área de secagem } & 2,0 \mathrm{~m}^{2} \\ \text { Dimensões (largura x altura x profundidade) } & 560 \times 930 \times 900 \mathrm{~mm} \\ \text { Consumo médio de gás } & 0,195 \mathrm{~kg} / \mathrm{h}\end{array}$

Um esquema do equipamento apresentando a disposição das bandejas, a posição do ventilador e a chama de aquecimento e circulação do ar está apresentado na Figura 3.

As curvas de secagem foram obtidas sob condições específicas no que se refere à velocidade do ar secante na superfície do produto, temperatura e umidade local, e não em relação a condições globais do equipamento como normalmente é feito. Mirade (2003) utilizou uma ferramenta computacional semelhante à utilizada neste trabalho para determinar as condições locais de velocidade e pressão em uma estufa de secagem de produtos cárneos. Foram medidas experimentalmente neste trabalho: a vazão global do ventilador na entrada do equipamento utilizando-se um anemômetro digital; a temperatura e umidade do ar de entrada utilizando-se um termo-higrômetro digital; além da temperatura na bandeja, na qual foram colocados os corpos-de-prova. Para determinar a velocidade do ar na bandeja

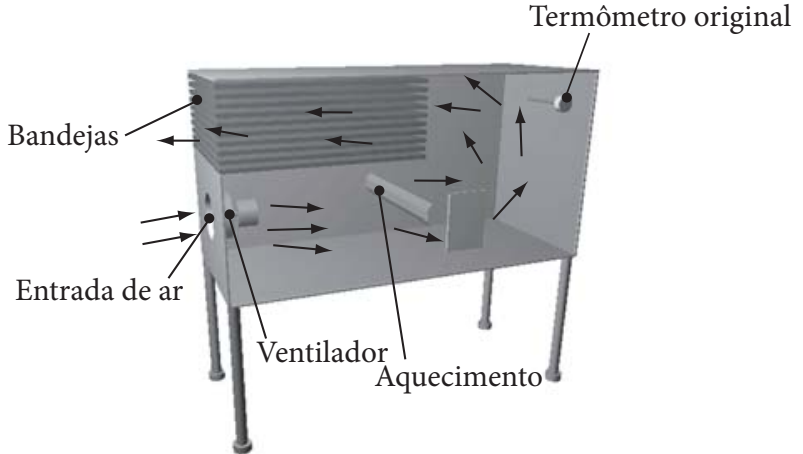

Figura 3. Esquema do equipamento de secagem.

foi utilizada uma ferramenta computacional para modelagem e simulação do escoamento do ar no interior da cavidade da estufa, conseguindo-se assim determinar as condições locais.

A simulação computacional foi realizada utilizando-se o programa Ansys, cujas equações governantes que descrevem o escoamento são resolvidas aplicando-se o método dos elementos finitos. As seguintes hipóteses foram consideradas:

- Escoamento em regime permanente;

- Simulação bidimensional no plano de simetria;

- Propriedades constantes do ar;

- Escoamento incompressível;

- Escoamento sem geração interna de calor; e

- Escoamento em plano de simetria.

Com isso, as equações governantes se reduzem a:

Equação da continuidade (Equação 1):

$\frac{\partial u}{\partial x}+\frac{\partial v}{\partial y}=0$

Equações de Navier-Stokes (Equação 2a e 2b):

$\rho\left(u \frac{\partial u}{\partial x}+v \frac{\partial u}{\partial y}\right)=-\frac{\partial p}{\partial x}+\mu\left(\frac{\partial^{2} u}{\partial x^{2}}+\frac{\partial^{2} u}{\partial y^{2}}\right)+\rho g_{x}$

$\rho\left(u \frac{\partial v}{\partial x}+v \frac{\partial v}{\partial y}\right)=-\frac{\partial p}{\partial y}+\mu\left(\frac{\partial^{2} v}{\partial x^{2}}+\frac{\partial^{2} v}{\partial y^{2}}\right)+\rho g_{y}$

Equação da Energia (Equação 3):

$\rho C_{p}\left(u \frac{\partial T}{\partial x}+v \frac{\partial T}{\partial y}\right)=k\left(\frac{\partial^{2} T}{\partial x^{2}}+\frac{\partial^{2} T}{\partial y^{2}}\right)+\mu \Phi$

em que $\Phi$ é dada por (Equação 4):

$\Phi=2\left[\left(\frac{\partial u}{\partial x}\right)^{2}+\left(\frac{\partial v}{\partial y}\right)^{2}\right]+\left(\frac{\partial v}{\partial x}+\frac{\partial u}{\partial y}\right)^{2}$ 
As condições de contorno consideradas estão apresentadas na Figura 4.

Numericamente foi considerada uma malha com as seguintes características: elementos triangulares lineares de três nós; 26469 elementos; 14357 nós; e a solução numérica foi considerada convergida se satisfeito o seguinte critério (Equação 5):

$$
\varepsilon=\frac{\sum_{i=1}^{n}\left|\varphi_{i}^{k}-\varphi_{i}^{k-1}\right|}{\sum_{i=1}^{n}\left|\varphi_{i}^{k}\right|} \leq 10^{-8}
$$

O trabalho de Bambirra et al. (2002) descreve a influência de quatro diferentes tipos de tratamentos pós-colheita na qualidade do açafrão, são eles: lavagem em água corrente, cozimento em água fervente por 90 minutos, cozimento em água alcalina contendo $0,1 \%$ de bicarbonato de sódio com $\mathrm{pH}$ de 8,6 , cozimento a $121^{\circ} \mathrm{C}$ por 40 minutos com e sem pele. Os resultados apontaram que a primeira alternativa de tratamento, que é a mais simples de todas, resultou no maior tempo de secagem, na maior umidade adquirida durante a estocagem e na maior perda de pigmento. Para o experimento realizado neste trabalho, os rizomas de açafrão não sofreram nenhum tipo de tratamento térmico pré-secagem, seguindo o mesmo procedimento adotado por Bambirra et al. (2002), mas diferindo nas formas geométricas do corte.

Com o objetivo de padronização dos experimentos, análise da influência da casca, relações do volume e massa com o tempo de secagem, além do fato de que os rizomas encontrados em sua forma natural não seguem um padrão geométrico definido, foram considerados dois tipos padrão de corpos-de-prova para análise, cujas características geométricas estão descritas a seguir na Tabela 3.

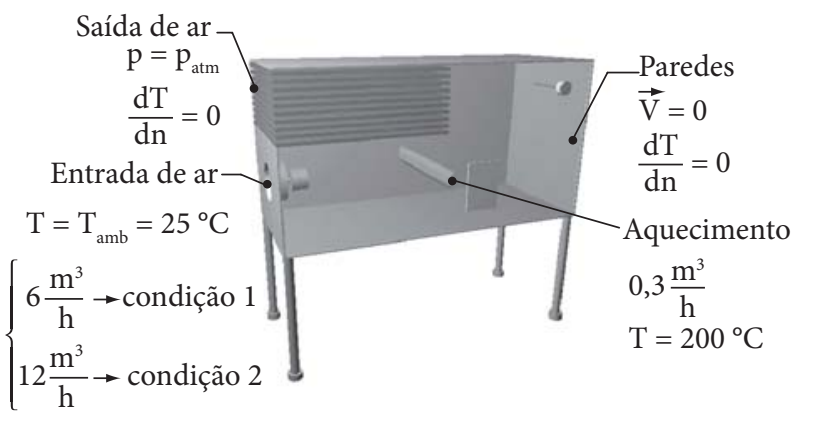

Figura 4. Condições de contorno para as equações de Navier-Stokes e energia.

Tabela 3. Características geométricas dos corpos-de-prova.

\begin{tabular}{ccc}
\hline Cubo com aresta $\mathrm{L}$ & Disco/cilindro com altura h e diâmetro d \\
\hline $\mathrm{L}=5 \mathrm{~mm}$ & $\mathrm{~h}=5 \mathrm{~mm}$ & $\mathrm{~d}=10 \mathrm{~mm}$ \\
$\mathrm{~L}=10 \mathrm{~mm}$ & $\mathrm{~h}=10 \mathrm{~mm}$ & $\mathrm{~d}=15 \mathrm{~mm}$ \\
$\mathrm{~L}=20 \mathrm{~mm}$ & $\mathrm{~h}=15 \mathrm{~mm}$ & $\mathrm{~d}=20 \mathrm{~mm}$ \\
& $\mathrm{~h}=20 \mathrm{~mm}$ & \\
\hline
\end{tabular}

As dimensões consideradas nas análises foram definidas segundo as definições de diâmetro médio e altura média (Equação 6 e Equação 7), e são definidas segundo Perry (2000) como uma média aritmética dos valores medidos de acordo com a Figura 5.

$\mathrm{d}_{\mathrm{m}}=\frac{\left(\mathrm{d}_{1}+\mathrm{d}_{2}\right)}{2}$

$\mathrm{h}_{\mathrm{m}}=\frac{\left(\mathrm{h}_{1}+\mathrm{h}_{2}\right)}{2}$

\section{Resultados e discussão}

As condições de escoamento no interior da estufa foram determinadas a partir da solução das equações de Navier-Stokes e da energia (Equações 1 a 4), utilizando o pacote computacional Ansys, e as curvas isobáricas, isotermas e linhas de corrente para as duas condições de secagem são apresentadas nas Figuras $6 a$, $6 \mathrm{~b}, 6 \mathrm{c}, 6 \mathrm{~d}, 6 \mathrm{e}$ e $6 \mathrm{f}$.

As velocidades locais, as temperaturas experimentais e calculadas numericamente na bandeja onde foram dispostos os corpos-de-prova estão apresentadas na Tabela 4.

Experimentalmente todos os corpos-de-prova foram dispostos em uma bandeja específica, conforme Figura 7, a sexta bandeja contada a partir da superior para a inferior, que foi escolhida anteriormente baseada em resultados preliminares de simulação computacional por estar na região onde há condições mais estáveis de escoamento.

As curvas de secagem para as diversas condições experimentais estão apresentadas na Figura 8, e a porcentagem de massa retirada foi calculada utilizando a Equação 8:

$$
\mathrm{m} \%=\left(\frac{\mathrm{m}_{\mathrm{i} \dot{\mathrm{i} 1} 1}-\mathrm{m}_{\mathrm{ath}}}{\mathrm{m}_{\mathrm{i} \dot{\mathrm{i}} 1}}\right) \times 100
$$

\subsection{Máxima quantidade de água retirada}

Após certo tempo, o produto atinge o seu equilíbrio com o meio secante, indicando assim o ponto onde a máxima quantidade de água pôde ser retirada sob aquelas condições específicas de secagem. Para os tempos considerados, pôde-se observar que para alguns dos corpos-de-prova o tempo foi suficientemente longo para que eles atingissem o equilíbrio com o meio, e para outros o tempo não foi suficiente. Para os casos em que foram atingidos os equilíbrios (ensaios 1, 2, 5, 6, 7 e 10), a quantidade máxima de água retirada foi próxima uma das outras, como pode-se verificar na Tabela 5, onde também estão em destaque os valores máximos e mínimos de equilíbrio para cada temperatura.
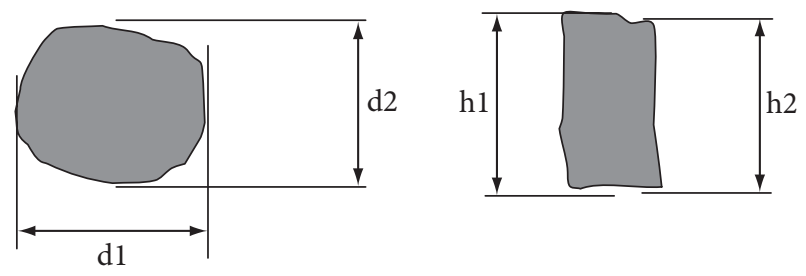

Figura 5. Diâmetro e altura média. 


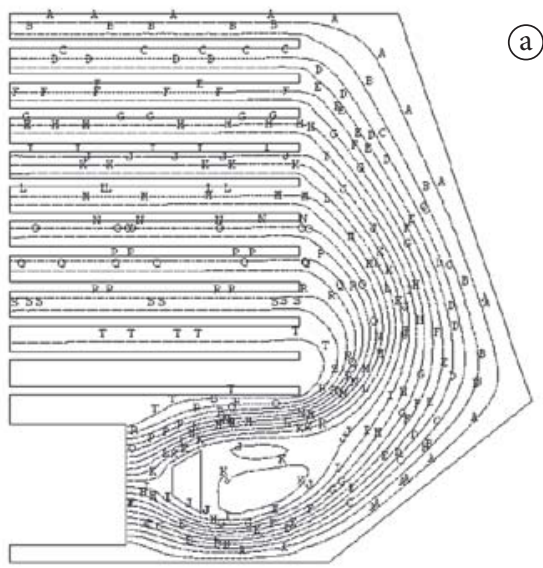

ANSYS 5.5.1

APR 72008 11:43:16

$\mathrm{A}=-.012155 \quad \mathrm{H}=-.007718 \quad \mathrm{O}=-.003282$

$\mathrm{B}=-.011521 \quad \mathrm{I}=-.007085 \quad \mathrm{P}=-.002648$

$\mathrm{C}=-.010888 \quad \mathrm{~J}=-.006451 \quad \mathrm{Q}=-.002014$

$\mathrm{D}=-.010254 \quad \mathrm{~K}=-.005817 \quad \mathrm{R}=-.00138$

$\mathrm{E}=-.00962 \quad \mathrm{~L}=-.005183 \quad \mathrm{~S}=-.746 \mathrm{E}-03$

$\mathrm{F}=-.008986 \quad \mathrm{M}=-.004549 \mathrm{~T}=-.113 \mathrm{E}-03$

$\mathrm{G}=-.008352 \quad \mathrm{~N}=-.003915$ (a)

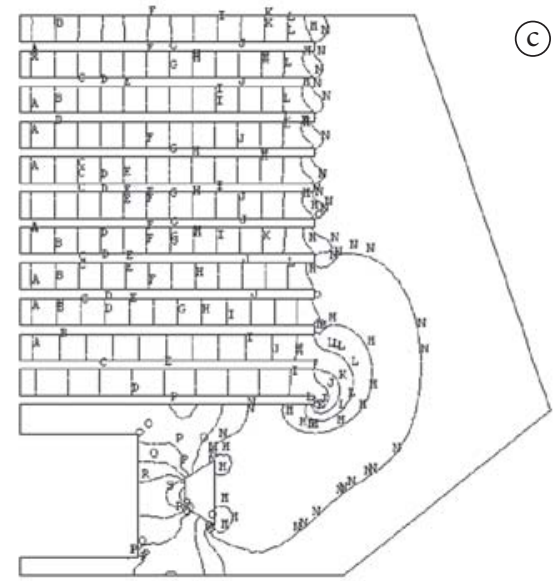

ANSYS 5.5.1

APR 72008 11:29:35

$\mathrm{A}=.522 \mathrm{E}-05 \quad \mathrm{H}=.783 \mathrm{E}-04 \quad \mathrm{O}=.151 \mathrm{E}-03$

$\mathrm{B}=.157 \mathrm{E}-04 \quad \mathrm{I}=.887 \mathrm{E}-04 \quad \mathrm{P}=.162 \mathrm{E}-03$

$\mathrm{C}=.261 \mathrm{E}-04 \quad \mathrm{~J}=.992 \mathrm{E}-04 \quad \mathrm{Q}=.172 \mathrm{E}-03$

$\mathrm{D}=.365 \mathrm{E}-04 \quad \mathrm{~K}=.110 \mathrm{E}-03 \quad \mathrm{R}=.183 \mathrm{E}-03$

$\mathrm{E}=.470 \mathrm{E}-04 \quad \mathrm{~L}=.120 \mathrm{E}-03 \quad \mathrm{~S}=.193 \mathrm{E}-03$

$\mathrm{F}=.574 \mathrm{E}-04 \quad \mathrm{M}=.130 \mathrm{E}-03 \quad \mathrm{~T}=.204 \mathrm{E}-03$

$\mathrm{G}=.679 \mathrm{E}-04 \quad \mathrm{~N}=.141 \mathrm{E}-03$

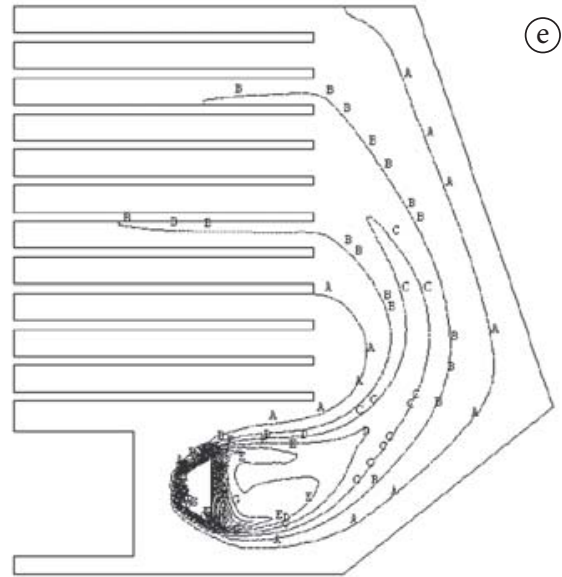

ANSYS 5.5.1

APR 72008 11:42:40

$\mathrm{A}=300.86 \quad \mathrm{H}=365.135 \quad \mathrm{O}=429.41$

$\mathrm{B}=310.042 \quad \mathrm{I}=374.317 \quad \mathrm{P}=438.592$

$\mathrm{C}=319.224 \quad \mathrm{~J}=383.499 \quad \mathrm{Q}=447.774$

$\mathrm{D}=328.406 \quad \mathrm{~K}=392.681 \quad \mathrm{R}=456.956$

$\mathrm{E}=337.588 \quad \mathrm{~L}=401.863 \quad \mathrm{~S}=466.139$

$\mathrm{F}=346.77 \quad \mathrm{M}=411.046 \mathrm{~T}=475.321$

$\mathrm{G}=355.953 \quad \mathrm{~N}=420.228$

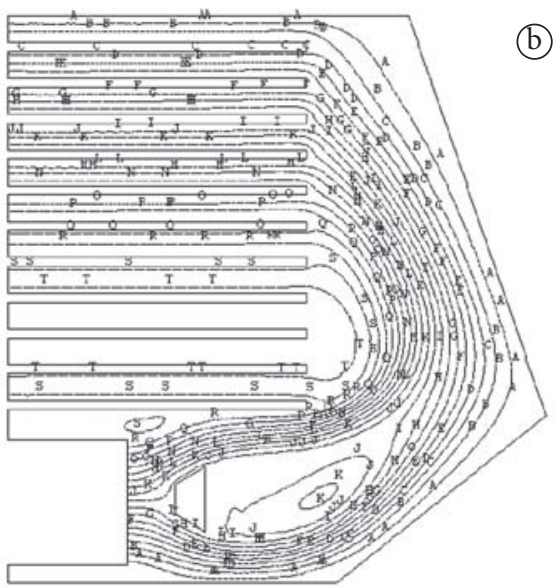

ANSYS 5.5.1

APR $72008 \quad 11: 46: 54$

$\mathrm{A}=-.024251 \quad \mathrm{H}=-.01476 \quad \mathrm{O}=-.005268$

$\mathrm{B}=-.022895 \quad \mathrm{I}=-.013404 \quad \mathrm{P}=-.003912$

$\mathrm{C}=-.021539 \quad \mathrm{~J}=-.012048 \quad \mathrm{Q}=-.002556$

$\mathrm{D}=-.020184 \quad \mathrm{~K}=-.010692 \quad \mathrm{R}=-.0012$

$\mathrm{E}=-.018828 \quad \mathrm{~L}=-.009336 \quad \mathrm{~S}=.156 \mathrm{E}-03$

$\mathrm{F}=-.017472 \quad \mathrm{M}=-.00798 \quad \mathrm{~T}=.001512$

$\mathrm{G}=-.016116 \quad \mathrm{~N}=-.006624$

(b)

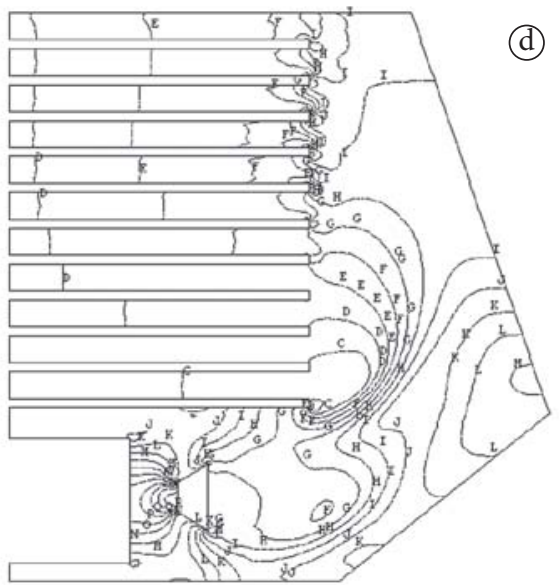

\section{ANSYS 5.5.1}

APR 72008 11:45:05

$\mathrm{A}=-004367 \quad \mathrm{H}=.006429$

$\mathrm{B}=-.002825 \quad \mathrm{I}=.007971$

$\mathrm{C}=-.001283 \quad \mathrm{~J}=.009513$

$\mathrm{D}=.260 \mathrm{E}-03 \quad \mathrm{~K}=.011056$

$\mathrm{E}=.001802 \quad \mathrm{~L}=.012598$

$\mathrm{F}=.003344 \quad \mathrm{M}=.01414$

$\mathrm{G}=.004886 \quad \mathrm{~N}=.015682$ (d)

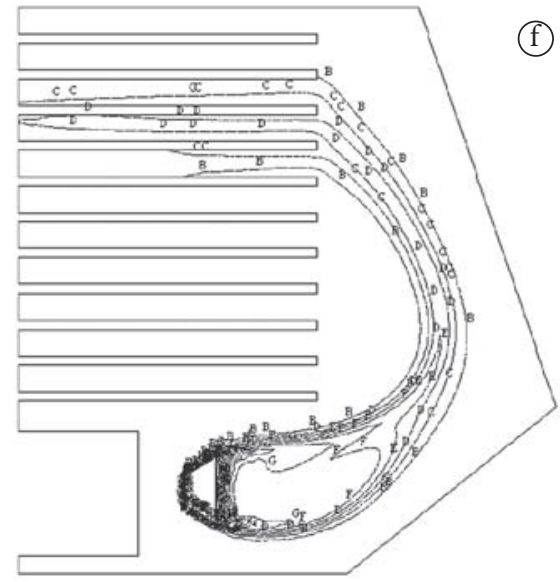

ANSYS 5.5.1

APR 72008 11:45:57

$\mathrm{A}=290.974 \quad \mathrm{H}=359.139 \quad \mathrm{O}=427.304$

$\mathrm{B}=300.712 \quad \mathrm{I}=368.877 \quad \mathrm{P}=437.042$

$\mathrm{C}=310.45 \quad \mathrm{~J}=378.615 \quad \mathrm{Q}=446.78$

$\mathrm{D}=320.188 \quad \mathrm{~K}=388.353 \quad \mathrm{R}=456.518$

$\mathrm{E}=329.926 \quad \mathrm{~L}=398.091 \quad \mathrm{~S}=466.256$

$\mathrm{F}=339.664 \quad \mathrm{M}=407.829 \quad \mathrm{~T}=475.994$

$\mathrm{G}=349.402 \quad \mathrm{~N}=417.567$

Figura 6. Linhas de corrente (a,b), isobáricas (c,d) e isotermas (e,f) $U_{e}=0,05 \mathrm{~m} / \mathrm{s}$ (esquerda) e $U_{e}=0,10 \mathrm{~m} / \mathrm{s}$ (direita).

Considerando a hipótese de que somente água é retirada durante o processo de secagem, podemos comparar os resultados obtidos da umidade do açafrão nos casos em que foram atingidos os equilíbrios com o meio secante, com valores conhecidos na literatura, conforme Tabela 6 .

\subsection{Influência da casca no tempo de secagem}

Nos ensaios 1, 2, 5, 6, 7 e 10, observou-se que em todos os casos o tempo foi suficiente para que fosse atingido o equilíbrio de umidade entre o produto e o ar secante, e nos demais casos isso não foi possível. 
Tabela 4. Valores locais de temperatura e velocidade.

\begin{tabular}{ccccccccc}
\hline Bandeja & \multicolumn{3}{c}{$\mathrm{U}_{\mathrm{e}}=0,05 \mathrm{~m} / \mathrm{s}$} & & \multicolumn{3}{c}{$\mathrm{U}_{\mathrm{e}}=0,10 \mathrm{~m} / \mathrm{s}$} \\
\cline { 2 - 4 } \cline { 7 - 9 } & $\mathrm{T}_{\text {exp }}[\mathrm{K}]$ & $\mathrm{T}_{\text {calc }}[\mathrm{K}]$ & $\mathrm{V}_{\text {média }}[\mathrm{m} / \mathrm{s}]$ & & $\mathrm{T}_{\text {exp }}[\mathrm{K}]$ & $\mathrm{T}_{\text {calc }}[\mathrm{K}]$ & $\mathrm{V}_{\text {média }}[\mathrm{m} / \mathrm{s}]$ \\
\hline 06 & 323,00 & 322,62 & $4,42 \mathrm{E}-02$ & & 316,00 & 317,19 & $1,05 \mathrm{E}-01$ \\
\hline
\end{tabular}

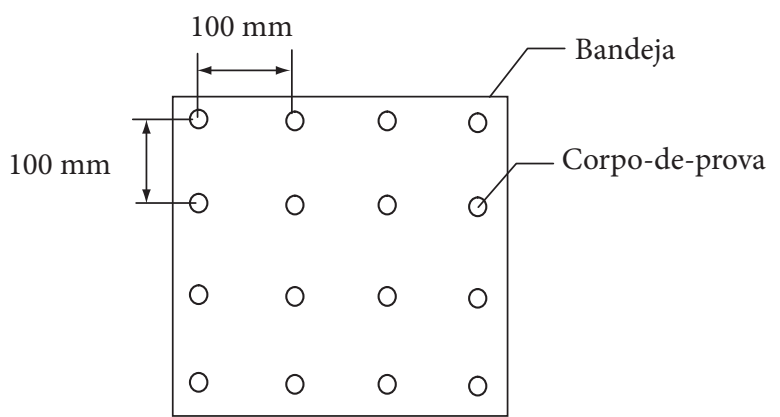

Tabela 6. Umidade relativa média de equilíbrio do açafrão.

\begin{tabular}{ll}
\hline \multicolumn{1}{c}{ Fonte } & $\mathrm{UR}(\%)$ \\
\hline Este trabalho $40^{\circ} \mathrm{C}\left(\mathrm{UR}_{\mathrm{ar}}=40 \%\right)$ & 10,85 \\
Este trabalho $50^{\circ} \mathrm{C}\left(\mathrm{UR}_{\mathrm{ar}}=\mathrm{t} 40 \%\right)$ & 10,22 \\
Leonel e Cereda $(2002) 105^{\circ} \mathrm{C}$ & 18,77 \\
Filho e Villas-Boas $(1996)$ & 25,30 \\
Bambirra et al. $(2002) 60^{\circ} \mathrm{C}$ & \\
Sem tratamento térmico/sem casca & 9,86 \\
Cozido sob pressão/sem casca & 9,20 \\
Cozido sob pressão/com casca & 8,93 \\
Cozido em água & 8,84 \\
Cozido em solução alcalina & 9,15 \\
\hline
\end{tabular}

Figura 7. Disposição dos corpos-de-prova na bandeja.

Tabela 5. Quantidade máxima de água retirada em porcentagem de peso.

\begin{tabular}{|c|c|c|c|c|c|c|c|c|c|}
\hline \multicolumn{5}{|c|}{$\mathrm{T}=40^{\circ} \mathrm{C}$} & \multicolumn{5}{|c|}{$\mathrm{T}=50^{\circ} \mathrm{C}$} \\
\hline \multicolumn{4}{|c|}{ Cilindro } & \multirow{2}{*}{$\begin{array}{c}\text { Cubo } \\
\text { Ensaio } 05\end{array}$} & \multicolumn{4}{|c|}{ Cilindro } & \multirow{2}{*}{$\begin{array}{c}\text { Cubo } \\
\text { Ensaio } 10\end{array}$} \\
\hline $\begin{array}{l}\text { Ensaio } 01 \\
\mathrm{~h}=5 \mathrm{~mm}\end{array}$ & $\begin{array}{c}\text { Ensaio } 02 \\
\mathrm{~h}=10 \mathrm{~mm}\end{array}$ & $\begin{array}{c}\text { Ensaio } 03 \\
\mathrm{~h}=15 \mathrm{~mm}\end{array}$ & $\begin{array}{c}\text { Ensaio } 04 \\
\mathrm{~h}=20 \mathrm{~mm}\end{array}$ & & $\begin{array}{l}\text { Ensaio } 06 \\
\mathrm{~h}=5 \mathrm{~mm}\end{array}$ & $\begin{array}{c}\text { Ensaio } 07 \\
\mathrm{~h}=10 \mathrm{~mm}\end{array}$ & $\begin{array}{c}\text { Ensaio } 08 \\
\mathrm{~h}=15 \mathrm{~mm}\end{array}$ & $\begin{array}{l}\text { Ensaio } 09 \\
\mathrm{~h}=20 \mathrm{~mm}\end{array}$ & \\
\hline $\begin{array}{c}\mathrm{d}=10 \mathrm{~mm} \\
91,32\end{array}$ & 88,42 & 77,98 & 59,24 & $\begin{array}{c}\mathrm{L}=5 \mathrm{~mm} \\
90,18\end{array}$ & $\begin{array}{c}\mathrm{d}=10 \mathrm{~mm} \\
92,10\end{array}$ & 89,36 & 89,67 & 88,05 & $\begin{array}{c}\mathrm{L}=5 \mathrm{~mm} \\
94,36\end{array}$ \\
\hline $\begin{array}{c}\mathrm{d}=15 \mathrm{~mm} \\
88,28\end{array}$ & 88,63 & 76,68 & 78,76 & $\begin{array}{c}\mathrm{L}=10 \mathrm{~mm} \\
86,93\end{array}$ & $\begin{array}{c}\mathrm{d}=15 \mathrm{~mm} \\
88,55\end{array}$ & 87,16 & 85,71 & 85,44 & $\begin{array}{c}\mathrm{L}=10 \mathrm{~mm} \\
87,22\end{array}$ \\
\hline $\begin{array}{c}\mathrm{d}=20 \mathrm{~mm} \\
90,39\end{array}$ & 90,16 & 78,56 & 77,16 & $\begin{array}{c}\mathrm{L}=20 \mathrm{~mm} \\
88,02\end{array}$ & $\begin{array}{c}\mathrm{d}=20 \mathrm{~mm} \\
90,65\end{array}$ & 90,26 & 86,99 & 86,47 & $\begin{array}{c}\mathrm{L}=20 \mathrm{~mm} \\
88,34\end{array}$ \\
\hline
\end{tabular}

Tabela 7. Porcentagem de área coberta com casca.

\begin{tabular}{|c|c|c|c|c|c|c|c|c|c|}
\hline \multicolumn{5}{|c|}{$\mathrm{T}=40^{\circ} \mathrm{C}$} & \multicolumn{5}{|c|}{$\mathrm{T}=50^{\circ} \mathrm{C}$} \\
\hline \multicolumn{4}{|c|}{ Cilindro } & \multirow{2}{*}{$\frac{\text { Cubo }}{\text { Ensaio } 05}$} & \multicolumn{4}{|c|}{ Cilindro } & \multirow{2}{*}{$\frac{\text { Cubo }}{\text { Ensaio } 10}$} \\
\hline $\begin{array}{l}\text { Ensaio } 01 \\
\mathrm{~h}=5 \mathrm{~mm}\end{array}$ & $\begin{array}{c}\text { Ensaio } 02 \\
\mathrm{~h}=10 \mathrm{~mm}\end{array}$ & $\begin{array}{c}\text { Ensaio } 03 \\
\mathrm{~h}=15 \mathrm{~mm}\end{array}$ & $\begin{array}{c}\text { Ensaio } 04 \\
\mathrm{~h}=20 \mathrm{~mm}\end{array}$ & & $\begin{array}{l}\text { Ensaio } 06 \\
\mathrm{~h}=5 \mathrm{~mm}\end{array}$ & $\begin{array}{c}\text { Ensaio } 07 \\
\mathrm{~h}=10 \mathrm{~mm}\end{array}$ & $\begin{array}{c}\text { Ensaio } 08 \\
\mathrm{~h}=15 \mathrm{~mm}\end{array}$ & $\begin{array}{c}\text { Ensaio } 09 \\
\mathrm{~h}=20 \mathrm{~mm}\end{array}$ & \\
\hline $\begin{array}{c}\mathrm{d}=10 \mathrm{~mm} \\
50,00\end{array}$ & 66,67 & 75,00 & 80,00 & $\begin{array}{c}\mathrm{L}=5 \mathrm{~mm} \\
0,00\end{array}$ & $\begin{array}{c}\mathrm{d}=10 \mathrm{~mm} \\
50,00\end{array}$ & 66,67 & 75,00 & 80,00 & $\begin{array}{c}\mathrm{L}=5 \mathrm{~mm} \\
0,00\end{array}$ \\
\hline $\begin{array}{c}\mathrm{d}=15 \mathrm{~mm} \\
39,76\end{array}$ & 57,08 & 66,67 & 72,68 & $\begin{array}{c}\mathrm{L}=10 \mathrm{~mm} \\
0,00\end{array}$ & $\begin{array}{c}\mathrm{d}=15 \mathrm{~mm} \\
39,76\end{array}$ & 57,08 & 66,67 & 72,68 & $\begin{array}{c}\mathrm{L}=10 \mathrm{~mm} \\
0,00\end{array}$ \\
\hline $\begin{array}{c}\mathrm{d}=20 \mathrm{~mm} \\
33,33\end{array}$ & 50,00 & 60,00 & 66,67 & $\begin{array}{c}\mathrm{L}=20 \mathrm{~mm} \\
0,00\end{array}$ & $\begin{array}{c}\mathrm{d}=20 \mathrm{~mm} \\
33,33\end{array}$ & 50,00 & 60,00 & 66,67 & $\begin{array}{c}\mathrm{L}=20 \mathrm{~mm} \\
0,00\end{array}$ \\
\hline
\end{tabular}

Tabela 8. Volume dos corpos-de-prova $\left[\mathrm{mm}^{3}\right]$.

\begin{tabular}{|c|c|c|c|c|c|c|c|c|c|}
\hline \multicolumn{5}{|c|}{$\mathrm{T}=40^{\circ} \mathrm{C}$} & \multicolumn{5}{|c|}{$\mathrm{T}=50^{\circ} \mathrm{C}$} \\
\hline \multicolumn{4}{|c|}{ Cilindro } & \multirow{2}{*}{$\begin{array}{c}\text { Cubo } \\
\text { Ensaio } 05\end{array}$} & \multicolumn{4}{|c|}{ Cilindro } & \multirow{2}{*}{$\begin{array}{c}\text { Cubo } \\
\text { Ensaio } 10\end{array}$} \\
\hline $\begin{array}{l}\text { Ensaio } 01 \\
\mathrm{~h}=5 \mathrm{~mm}\end{array}$ & $\begin{array}{c}\text { Ensaio } 02 \\
\mathrm{~h}=10 \mathrm{~mm}\end{array}$ & $\begin{array}{l}\text { Ensaio } 03 \\
\mathrm{~h}=15 \mathrm{~mm}\end{array}$ & $\begin{array}{r}\text { Ensaio } 04 \\
\mathrm{~h}=20 \mathrm{~mm}\end{array}$ & & $\begin{array}{l}\text { Ensaio } 06 \\
\mathrm{~h}=5 \mathrm{~mm}\end{array}$ & $\begin{array}{l}\text { Ensaio } 07 \\
\mathrm{~h}=10 \mathrm{~mm}\end{array}$ & $\begin{array}{l}\text { Ensaio } 08 \\
\mathrm{~h}=15 \mathrm{~mm}\end{array}$ & $\begin{array}{r}\text { Ensaio } 09 \\
\mathrm{~h}=20 \mathrm{~mm}\end{array}$ & \\
\hline $\begin{array}{c}\mathrm{d}=10 \mathrm{~mm} \\
392,00\end{array}$ & 785,00 & 1178,00 & 1570,00 & $\begin{array}{c}\mathrm{L}=5 \mathrm{~mm} \\
1000,00\end{array}$ & $\begin{array}{c}\mathrm{d}=10 \mathrm{~mm} \\
392,00\end{array}$ & 785,00 & 1178,00 & 1570,00 & $\begin{array}{c}\mathrm{L}=5 \mathrm{~mm} \\
1000,00\end{array}$ \\
\hline $\begin{array}{c}\mathrm{d}=15 \mathrm{~mm} \\
883,00\end{array}$ & 1767,00 & 2650,00 & 3534,00 & $\begin{array}{c}\mathrm{L}=10 \mathrm{~mm} \\
3375,00\end{array}$ & $\begin{array}{c}\mathrm{d}=15 \mathrm{~mm} \\
883,00\end{array}$ & 1767,00 & 2650,00 & 3534,00 & $\begin{array}{c}\mathrm{L}=10 \mathrm{~mm} \\
3375,00\end{array}$ \\
\hline $\begin{array}{c}\mathrm{d}=20 \mathrm{~mm} \\
1570,00\end{array}$ & 3141,00 & 4712,00 & 6283,00 & $\begin{array}{c}\mathrm{L}=20 \mathrm{~mm} \\
8000,00\end{array}$ & $\begin{array}{c}\mathrm{d}=20 \mathrm{~mm} \\
1570,00\end{array}$ & 3141,00 & 4712,00 & 6283,00 & $\begin{array}{c}\mathrm{L}=20 \mathrm{~mm} \\
8000,00\end{array}$ \\
\hline
\end{tabular}

Tabela 9. Tempos de equilíbrio na secagem do açafrão.

\begin{tabular}{|c|c|c|c|}
\hline $\begin{array}{l}\text { Este trabalho } \\
\text { Menor tempo de equilíbrio }\end{array}$ & $\begin{array}{l}\text { Este trabalho } \\
\text { Maior tempo de equilíbrio }\end{array}$ & \multicolumn{2}{|c|}{$\begin{array}{l}\text { Bambirra et al. (2002) } \\
\text { Tempo médio de secagem }\end{array}$} \\
\hline $\begin{array}{c}\text { Cubo, } \mathrm{L}=5 \mathrm{~mm}, \mathrm{~T}=50^{\circ} \mathrm{C}, \\
\text { Vsup }=4,42 \mathrm{E}-02 \mathrm{~m} / \mathrm{s}\end{array}$ & $\begin{array}{c}\text { Cubo, } \mathrm{L}=20 \mathrm{~mm}, \mathrm{~T}=40^{\circ} \mathrm{C} \\
\text { Vsup }=1,05 \mathrm{E}-01 \mathrm{~m} / \mathrm{s}\end{array}$ & Sem tratamento térmico & Com tratamento térmico \\
\hline 10 horas & 200 horas & 168 horas & 144 horas \\
\hline
\end{tabular}




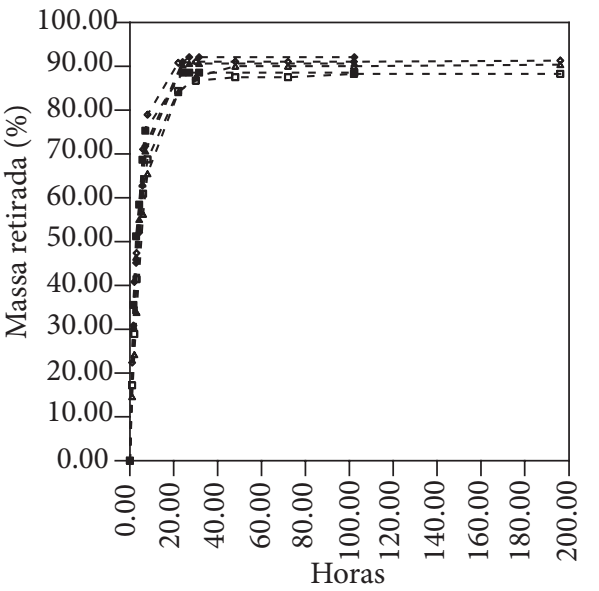

Disco h $=5 \mathrm{~mm}$

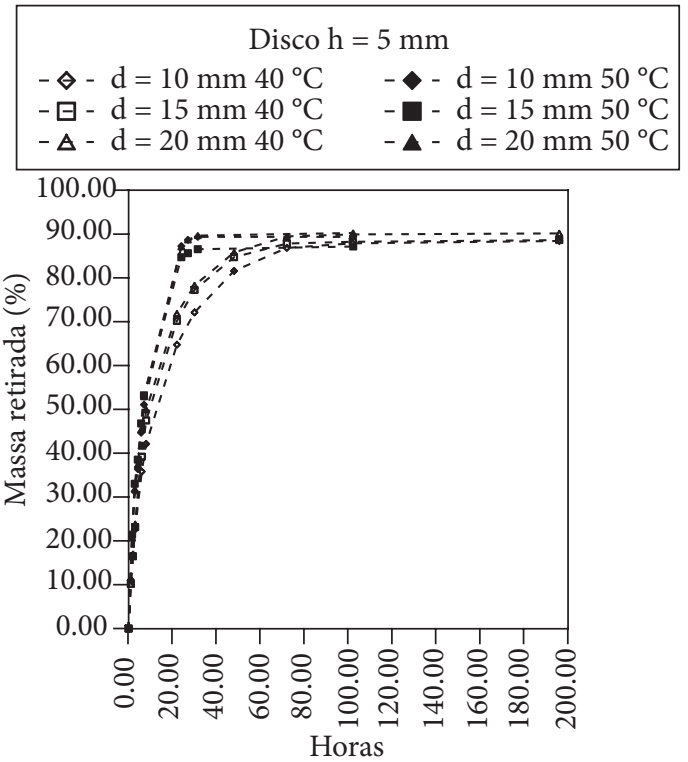

Disco h $=10 \mathrm{~mm}$

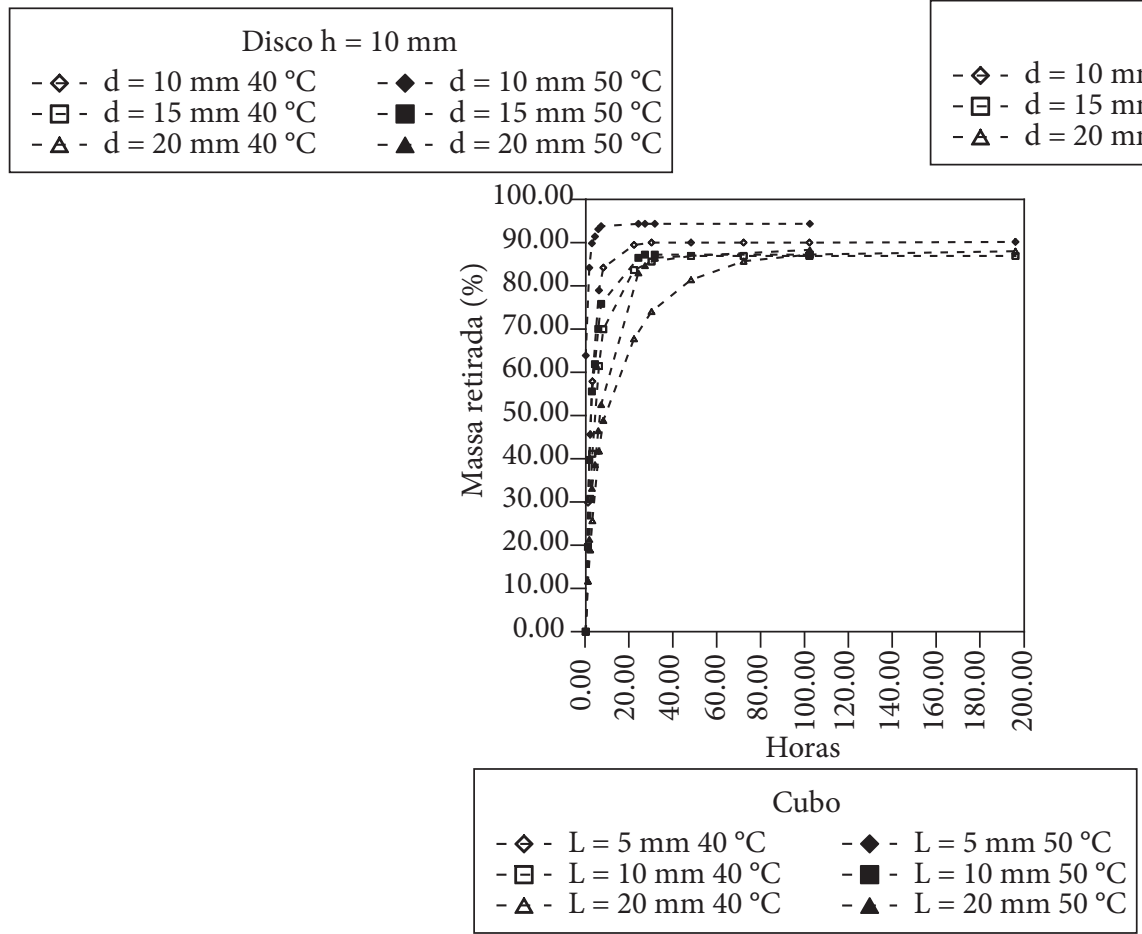

Figura 8. Quantidade de massa retirada em função do tempo de secagem.

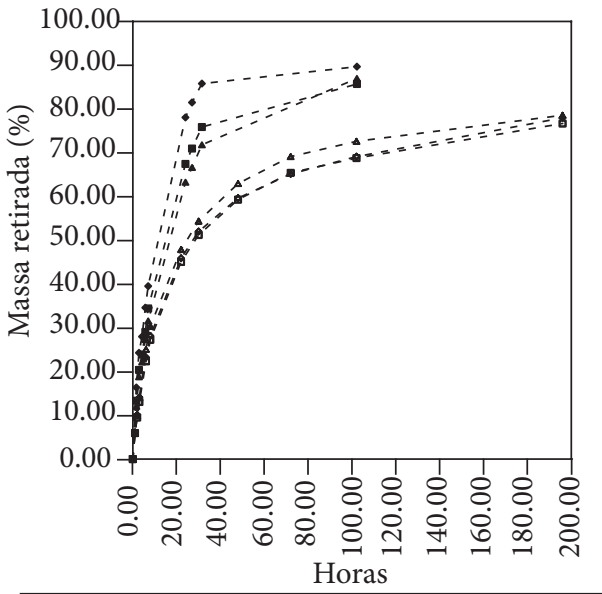

Disco $\mathrm{h}=15 \mathrm{~mm}$
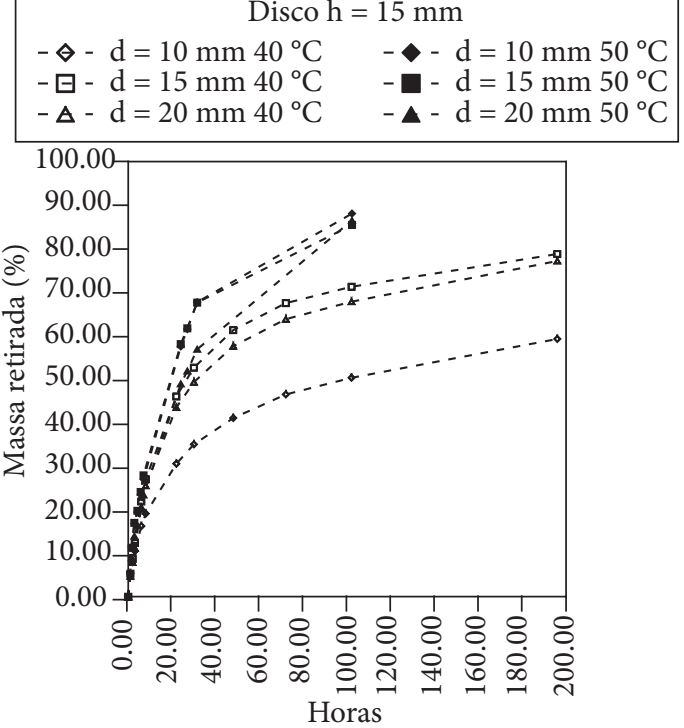

Disco $\mathrm{h}=20 \mathrm{~mm}$

$\diamond-\mathrm{d}=10 \mathrm{~mm} 40{ }^{\circ} \mathrm{C} \quad-\diamond-\mathrm{d}=10 \mathrm{~mm} 50^{\circ} \mathrm{C}$

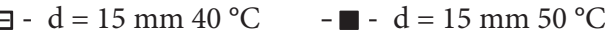
- $\boldsymbol{\Delta}-\mathrm{d}=20 \mathrm{~mm} 50^{\circ} \mathrm{C}$ 
Os corpos-de-prova dos ensaios 1, 2, 6 e 7 são na forma de disco, cuja relação entre as áreas com casca e sem casca é baixa, pois como têm altura h menor que as demais, a difusão dificultada pela área com casca é compensada pela área sem casca. Contudo, ocorre o contrário para os casos dos ensaios 3, 4, 8 e 9, cujas porcentagens de área com casca são maiores, como apresentadas na Tabela 7.

Para os ensaios 5 e 10 esta relação não foi calculada, pois o corpo-de-prova é da forma cúbica e sem casca. Sabe-se que tanto o volume quanto a forma geométrica do corpo-de-prova influenciam na difusão, e isto pôde ser observado nas diferenças entre os tempos de secagem dos ensaios acima citados, o que confirma ainda mais a influência da casca na secagem, pois em corpos com volumes aproximados, cúbicos e cilíndricos pôde-se notar que os cúbicos sem casca atingiram o equilíbrio de umidade e os cilíndricos com casca não atingiram o equilíbrio. $\mathrm{O}$ volume de cada corpo-de-prova está apresentado na Tabela 8.

Dos resultados apresentados, ainda podemos identificar os menores e maiores tempos de secagem necessários para atingir o equilíbrio em cada situação, de acordo com a Tabela 9.

\section{Conclusões}

Com base nos gráficos apresentados, pôde-se levantar informações importantes para o processo de secagem do açafrão, tais como: tempo, cinética do processo, umidade de equilíbrio e influência da casca no processo. Os resultados se mostraram bem próximos dos valores disponíveis na literatura, no que se refere à umidade relativa de equilíbrio e tempos de secagem.

A relação de tempo e temperatura pôde ser verificada claramente nos gráficos das curvas de secagem apresentados. Observou-se que para um aumento de aproximadamente $10{ }^{\circ} \mathrm{C}$ na temperatura média de secagem, em geral o tempo foi reduzido em aproximadamente $45 \%$, o que demonstra a grande influência desta variável para o processo, sendo esta mais significativa que a variação da velocidade do ar secante, fenômeno também observado por Peña e Kieckbusch (2003) no processo de secagem de tomates.

A utilização da ferramenta computacional Ansys foi muito importante para determinação das condições de escoamento, velocidade e temperatura na superfície de troca do produto.

Por esta discussão apresentada, pode-se sugerir que para uma secagem mais rápida, e conseqüentemente, com menor gasto de energia, é importante que os cortes de açafrão tenham a menor quantidade de casca possível, pois esta dificulta na difusão da umidade para o ambiente.

\section{Referências bibliográficas}

ARAÚJO, C. A. C.; LEON, L. L. "Biological Activities of Curcuma longa L". Memórias do Instituto Osvaldo, Cruz Mem. Inst. Oswaldo Cruz, Rio de Janeiro, v. 96, n. 5, p. 723-728, July 2001.

BAMBIRRA, M. L. A.; JUNQUEIRA, R. G.; GLÓRIA, M. B. A. "Influence of Post Harvest Processing Conditions on Yield and Quality of Ground Turmeric (Curcuma longa L.)". Brazilian Archives of Biology and Technology, Curitiba, v. 45, n. 4, p. 423-429, Dec., 2002.

Brace Research Institute, MacDonald College of McGill University. A Survey of Solar Agricultural Dryers. Technical Report, Quebec, Canada, n. T99, Dec., 1975.

FILHO, A. B. C. et al. "Cúrcuma: Planta Medicinal, Condimentar e de Outros Usos Potenciais”. Ciência Rural, Santa Maria, v. 30, n. 1, p. $171-175,2000$.

FOGGIATO, E. C.; RIBANI, R. H. “Determinação do Teor de Umidade do Feijão Preto Utilizando Aparelho de Microondas". In: Congresso Brasileiro de Ciência e Tecnologia de Alimentos, 17, Fortaleza, 2000. Anais... Fortaleza, 2000.

KUHN, O. J. et al. "Efeito do Extrato Aquoso de Cúrcuma (Curcuma longa) em Xanthomonas axonopodis pv. manihotis". Seminário Ciências Agrárias, Londrina, v. 27, n. 1, p. 1-20, 2006.

MASKAN, M. "Kinetics of Colour Change of Kiwifruits During Hot Air and Microwave Drying". Journal of Food Engineering, v. 48, n. 2, p. 169-175, May 2001.

MASKAN, M. "Microwave/Air and Microwave Finish Drying of Banana". Journal of Food Engineering, v. 44, n. 2, p. 71-78, May 2000.

MIRADE, P. S. "Prediction of the Air Velocity Field in Modern Meat Dryers Using Unsteady Computational Fluid Dynamics (CFD) Models". Journal of Food Engineering, v. 60, n. 1, p. 41-48, November 2003.

PEÑA, L. M. R.; KIECKBUSCH, T. G. "Influência de Condições de Secagem na Qualidade de Fatias de Tomate". Brazilian Journal of Food Technology, Campinas, v. 6, n. 1, p. 69-76, 2003.

PEÑA, M. I. B. et al. "Controle de Alternaria solani em Tomateiro por Extratos de Curcuma longa e Curcumina - I. Avaliação in vitro". Fitopatologia Brasileira, v. 31, n. 3, p. 310-314, maio/jun, 2006a.

PEÑA, M. I. B. et al. "Controle de Alternaria solani em Tomateiro por Extratos de Curcuma longa e Curcumina - II. Avaliação in vivo”. Fitopatologia Brasileira, v. 31, n. 4, p. 401-404, jul/ago, 2006 b.

PERRY, R. H. "Perry's Chemical Engineer's Handbook". 7 ed., New York Local: Mc Graw Hill, 2000.

ROSSI, S. J.; ROA, G. "Secagem e Armazenamento de Produtos Agropecuários com o Uso de Energia Solar e Ar Natural". Publicação ACIESP, n. 22, 1980.

VALENTINI, S. R. T.; CASTRO, M. F. P. M.; ALMEIDA, F. H. "Determinação do Teor de Umidade de Milho Utilizando Aparelho de Microondas". Ciência e Tecnologia de Alimentos, Campinas, v. 18, n. 2, Maio, 1998. 